\title{
EXISTENCIA Y UNICIDAD DE SOLUCIONES DE ECUACIONES DIFERENCIALES FRACCIONARIAS
}

Pucheta, Pablo I. ${ }^{1}$

\begin{abstract}
Resumen
En este trabajo estudiaremos el problema de la existencia y la unicidad de la solución de ecuaciones diferenciales no lineales de orden fraccionario, usando la derivada de Caputo como el operador diferencial. Abordaremos esta cuestión mediante una equivalencia entre una ecuación diferencial fraccionaria y una ecuación integral de Volterra de segunda clase.
\end{abstract}

Palabras Claves: Ecuación Integral de Volterra, Cálculo Fraccionario y Transformada de Laplace.

\section{Introducción}

Es conocida la importancia de las ecuaciones diferenciales en la modelización de fenómenos estudiados por diversas ciencias. Al ser concebido el Cálculo fraccionario como una generalizacion del Análisis Matemático, es natural encontrar en su ámbito estudios sobre ecuaciones diferenciales fraccionarias análogos a los realizados sobre ecuaciones diferenciales ordinarias que pueden ser vistos como correspondientes unos de otros, pero tambíen surgen diferencias como ser las posibles interpretaciones físicas segun el tipo de derivada fraccionaria que se utilice.

El proposito de este artículo, que reune los resultados que formó parte de la tesis de Licenciatura en Matemática de Pablo I. Pucheta bajo la dirección del Dr. Ruben A. Cerruti, es solucionar el problema de la existencia y la unicidad de ecuaciones diferenciales fraccionarias mediante una equivalencia con una ecuación de Volterra de segunda clase.

Antes de comenzar es necesrio realizar una revisón de conceptos elementales que luego utilizaremos a lo largo de este artículo.

1. Facultad de Ciencias Exactas y Naturales y Agrimensura. Av. Libertad 5540 (3400); Corrientes, ARGENTINA Email: pablo.pucheta@hotmail.com 


\section{Preliminares}

En esta sección se darán las definiciones de funciones que son muy importantes en la teoría del cálculo fraccionario a saber, la función Gamma de Euler, la función Beta y la función de Mittag-Leffler como así también la relación entre la función gamma y la función beta.

\subsection{Ecuaciones Integrales}

Una ecuación integral se define como una ecuación funcional que contiene a la función incognita dentro del signo de la integral.

$$
\Phi(x)=f(x)+\int_{a}^{b} K(x, t) \Phi(t) d t
$$

donde $f$ y $K$ son funciones conocidas y $\Phi$ es la función incógnita. (cf.[?])

Dentro de la teória clásica de las ecuaciones integrales, por un lado tenemos las ecuaciones integrales de Fredholm y por otro las ecuaciones integrales de Volterra. En las ecuaciones integrales de Fredholm la región de integración es fija, tal como(1.2.1), mientras que en las ecuaciones integrales de Volterra la región de integración es variable.

Sea $I=[0, T]$ un intervalo cerrado y acotado, con $T>0$ y $S=\{(x, s): 0 \leq s \leq x \leq T\}$. La ecuación integral de Volterra más general viene dada por

$$
\Psi(x) \Phi(x)=f(x)+\int_{0}^{x} K(x, s, \Phi(s)) d s
$$

donde $\Psi, f, \Phi: I \rightarrow \mathbb{R}$ son funciones continuas y $K: S \times \mathbb{R} \rightarrow \mathbb{R}$ es el núcleo de la ecuación integral.

\subsection{Función Gamma}

La función Gamma de Euler generaliza el concepto de factorial de un número $n$, $n>0$ y permite tomar valores no enteros e incluso valores complejos.

La Función Gamma, se denota por $\Gamma$ y es definida por la integral

$$
\Gamma(z)=\int_{0}^{\infty} e^{-t} t^{z-1} d t
$$

la cual converge en el semiplano complejo $\operatorname{Re}(z)>0$ (cf.[8])

Una de las propiedades de la Función Gamma es la siguiente:

$$
\Gamma(z+1)=z \Gamma(z)
$$

la cual se demuestra, usando intregracion por partes en (2.3). En particular si $n \in \mathbb{N}$ se obtiene: $\Gamma(n+1)=n \Gamma(n)=n(n-1) !=n$ ! 


\subsection{Función Beta}

La función Beta se define mediante la integral

$$
B(z, w)=\int_{0}^{1} s^{z-1}(1-s)^{w-1} d s \quad(\operatorname{Re}(z)>0, \operatorname{Re}(w)>0) s \in \mathbb{R}
$$

Esta función esta ligada con la función gamma por la siguiente relación:

$$
B(z, w)=\frac{\Gamma(z) \Gamma(w)}{\Gamma(z+w)}
$$

\subsection{Función de Mittag-Leffler}

La función exponencial $e^{z}$ juega un papel importante en las soluciones de ecuaciones diferenciales de orden entero, por eso es lógico esperar que en las ecuaciones diferenciales fraccionarias exista una función que desempeñe ese rol similar, tal es así que esa función es la Función de Mittag-Leffler que generaliza la función exponencial. Para lo que sigue ver $([8])$

La función de Mittag-Leffler se define como

$$
E_{\alpha}(z)=\sum_{k=0}^{\infty} \frac{z^{k}}{\Gamma(\alpha k+1)} \quad z \in \mathbb{C}, \quad \alpha>0
$$

y la función de Mittag-Leffler de dos parametros se define como

$$
E_{\alpha, \beta}(z)=\sum_{k=0}^{\infty} \frac{z^{k}}{\Gamma(\alpha k+\beta)} \quad \alpha, \beta>0
$$

Notemos que sí $\alpha=1$ entonces:

- Sí $\beta=1$

$$
E_{1,1}(z)=\sum_{k=0}^{\infty} \frac{z^{k}}{\Gamma(1 k+1)}=\sum_{k=0}^{\infty} \frac{z^{k}}{\Gamma(k+1)}=\sum_{k=0}^{\infty} \frac{z^{k}}{k !}=e^{z}
$$

- Sí $\beta=2$

$$
E_{1,2}(z)=\sum_{k=0}^{\infty} \frac{z^{k}}{\Gamma(k+2)}=\sum_{k=0}^{\infty} \frac{z^{k}}{(k+1) !}=\sum_{k=0}^{\infty} \frac{z^{k+1}}{z} \frac{1}{(k+1) !}=\frac{e^{z}-1}{z}
$$




\section{Operadores de Derivación e Integración Frac- cionarios}

En esta sección se dan las definiciones de ciertos operadores fraccionarios. Se muestran algunas propiedades de estos operadores que nos sirven de base para la próxima seccíon ver (cf.[8])

\subsection{Integral Fraccionaria de Riemann-Liouville}

Definición 1 Sean $f \in L^{1}[a, b]$ y $\alpha \in \mathbb{R}^{+}$. La integral fraccionaria de Riemann-Liouville de orden $\alpha>0$ es definida por:

$$
I_{a}^{\alpha} f(x)=\frac{1}{\Gamma(\alpha)} \int_{a}^{x}(x-s)^{\alpha-1} f(s) d s \quad a<x<b
$$

1. Sí $\alpha<1$ la integral en (2.1.1) es impropia

2. Sí $\alpha \leq 0$ la integral diverge

3. Sí $0<\alpha<1$, la integral impropia converge

Dos propiedades importante de la integral fraccionaria de Riemann-Liouville son (cf.[8])

1. Bajo ciertas condiciones impuestas sobre $f$ se tiene que

$$
\lim _{\alpha \rightarrow 0} I_{a}^{\alpha} f(x)=f(x)
$$

2. Sean $\alpha, \mu \in \mathbb{R}^{+}$y $f \in L^{1}[a, b]$. Entonces

$$
I_{a}^{\alpha}\left(I_{a}^{\mu} f(x)\right)=I_{a}^{\alpha+\mu} f(x)
$$

\subsection{Derivada Fraccionaria de Riemann-Liouville}

Definición 2 Sea $\alpha \in \mathbb{R}^{+} y m-1 \leq \alpha<m$. La derivada fraccionaria de orden $\alpha$ de una función $f$ es definida por:

$$
D_{a}^{\alpha} f(x)=\frac{d^{m}}{d x^{m}}\left[\frac{1}{\Gamma(m-\alpha)} \int_{a}^{x}(x-t)^{m-\alpha-1} f(t) d t\right]=D^{m}\left(I_{a}^{m-\alpha} f(x)\right)
$$

Para $\alpha=k$ con $k \in \mathbb{N}$ y $x \geq a$ y (3.2) se tiene que

$$
D_{a}^{\alpha} f(x)=D^{k}\left(I_{a}^{0} f(x)\right)=D^{k} f(x)
$$

Por lo tanto, la derivada fraccionaria de Riemann-Liouville de orden $\alpha=m$ coicide con la derivada convencional de orden $m$

Dos propiedades importantes de la derivada fraccionaria de Riemann-Liouville son (cf.[8]): 
1. Sean $\alpha>0$ y $x>a$. Si $f \in L^{1}[a, b]$, entonces

$$
D_{a}^{\alpha}\left(I_{a}^{\alpha} f(x)\right)=f(x)
$$

2. Sean $n-1 \leq \alpha<n, n \in \mathbb{N}$ y $f \in L^{1}[a, b]$. Entonces

$$
I_{a}^{\alpha}\left(D_{a}^{\alpha} f(x)\right)=f(x)-\sum_{i=1}^{n}\left[D_{a}^{\alpha-i} f(x)\right]_{x=a} \frac{(x-a)^{\alpha-i}}{\Gamma(\alpha-i+1)}
$$

\subsection{Derivada Fraccionaria de Caputo}

En esta parágrafo vamos a dar otra definición de derivada fraccionaria de orden $\alpha$; Derivada Fraccionaria de Caputo y se enunciarán algunas propiedades de este nuevo operador. Además se establecerá una importante relación entre la Derivada Fraccionaria de Riemann-Liouville y la Derivada Fraccionaria de Caputo.

Definición 3 Sea $\alpha \in \mathbb{R}, m-1 \leq \alpha<m$. La derivada fraccionaria de Caputo de orden $\alpha$ de una función $f$, se denota como $D_{* a}^{\alpha}$ y es definida por

$$
D_{* a}^{\alpha} f(x)=\frac{1}{\Gamma(m-\alpha)} \int_{a}^{x}(x-t)^{m-\alpha-1} \frac{d^{m}}{d t^{m}} f(t) d t=I_{a}^{m-\alpha} D^{m} f(x)
$$

para $a \leq x \leq b$

Veamos ahora una importante relación entre la derivada fraccionaria de RiemannLiouville con la derivada fraccionaria de Caputo.(cf.[8])

Lema 1 Sean $\alpha \geq 0$ y $\alpha=\lceil m\rceil$ (parte entera). Supongamos que $f \in A C^{m}[a, b]$. Entonces

$$
D_{* a}^{\alpha} f(x)=D_{a}^{\alpha}\left[f-T_{m-1}[f ; a]\right]
$$

en casi todas partes, donde $T_{m-1}[f ; a]$ denota el polinomio de Taylor de grado $m-1$ para la función $f$, centrado en a. Osea

$$
T_{m-1}[f ; a](x)=\sum_{i=0}^{m-1} \frac{f^{(i)}(a)}{i !}(x-a)^{i}
$$

Observación. 1 Sí $f$ es una función para la cual $D_{* a}^{\alpha} f$ y $D_{a}^{\alpha} f$ existen, con $\alpha \in \mathbb{R}^{+}$, además $D^{i} f(a)=0, i=0,1, \ldots \ldots, m-1$. Entonces

$$
D_{* a}^{\alpha} f(x)=D_{a}^{\alpha} f(x)
$$

En efecto, teniendo en cuenta la linealidad de la derivada fraccionaria de RiemannLiouville y (3.9),(3.10) se tiene que

$$
\begin{aligned}
D_{* a}^{\alpha} f(x) & =D_{a}^{\alpha}\left[f-T_{m-1}[f ; a]\right](x) \\
& =D_{a}^{\alpha} f(x)-\underbrace{D_{a}^{\alpha} T_{m-1}[f ; a](x)}_{D}
\end{aligned}
$$




$$
\begin{aligned}
D=D_{a}^{\alpha} T_{m-1}[f ; a](x) & =D_{a}^{\alpha}\left(\sum_{i=0}^{m-1} \frac{f^{(i)}(a)}{i !}(x-a)^{i}\right) \\
& =\sum_{i=0}^{m-1} \frac{f^{(i)}(a)}{i !} D_{a}^{\alpha}(x-a)^{i} \\
& =\sum_{i=0}^{m-1} \frac{D^{(i)} f(a)}{i !} \frac{\Gamma(i+1)}{\Gamma(i-\alpha+1)}(x-a)^{i-\alpha} \\
& =\sum_{i=0}^{m-1} \frac{D^{(i)} f(a)}{\Gamma(i-\alpha+1)}(x-a)^{i-\alpha}
\end{aligned}
$$

Luego

$$
D_{* a}^{\alpha} f(x)=D_{a}^{\alpha} f(x)-\sum_{i=0}^{m-1} \frac{D^{(i)} f(a)}{\Gamma(i-\alpha+1)}(x-a)^{i-\alpha}
$$

\section{Existencia y Unicidad de la Solución de una Ecuación Diferencial Fraccionaria}

En este sección, se tratará la teoría sobre la existencia y unicidad de la solución del Problema de Valor Inicial asociado a las ecuaciones diferenciales ordinarias de orden fraccionario del tipo $D_{a}^{\alpha} f(x)=f((x, y(x))$ con la derivada de Caputo, con condiciones iniciales adecuadas. Para las definiciones siguientes ver (cf.[1])

Definición 4 Una ecuación diferencial de orden fraccionario $\alpha_{k}$, siendo $\alpha_{k}$ un número real es una relación del tipo

$$
F\left(x, y(x), D_{a}^{\alpha_{1}} y(x), \ldots \ldots \ldots, D_{a}^{\alpha_{k}} y(x)\right)=g(x)
$$

donde $F\left(x, y_{1}(x), \ldots \ldots, y_{n}(x)\right)$ y $g(x)$ son funciones reales conocidas y $D_{a}^{\alpha_{k}}$ son operadores diferenciales fraccionariios con $\alpha_{k}>0$ y $\alpha_{1}<\alpha_{2}<\ldots \ldots<\alpha_{k}$

Definición 5 Se denomina solución de una ecuación diferencial fraccionaria a cualquier función $y(x)$ que verifique la igualdad (3.0.1)

Definición 6 Diremos que una ecuación diferencial fraccionaria es lineal si tiene la siguiente forma

$$
\phi_{0}(x) y(x)+\sum_{i=1}^{m-1} \phi_{i}(x) D_{a}^{\alpha_{i}} y(x)=g(x)
$$

donde $\phi_{i}(x),(i=1, \ldots \ldots, m-1), g(x)$ son funciones conocidas y $D_{a}^{\alpha_{i}},(i=1, . ., m-1)$ son los operadores fraccionarios. 
Definición 7 1. Cuando se cumple que las funciones $\phi_{i}(x),(i=1, \ldots, m-1)$ y $g(x)$ son constantes, diremos que la ecuación diferencial fraccionaria es de coeficientes constantes.

2. Si se cumple que $g(x)=0$, diremos que la ecuación diferencial fraccionaria lineal es homogenea.

Definición 8 Sea $\alpha \in \mathbb{R}^{+} n=\lceil\alpha\rceil+1$ y $g: A \subseteq \mathbb{R}^{2} \rightarrow \mathbb{R}$. Entonces, la ecuación

$$
D_{* 0}^{\alpha} f(x)=g(x, f(x))
$$

sujeta a las $n$ condiciones iniciales

$$
f^{(i)}(0)=b_{i},(i=0,1, \ldots, n-1)
$$

es llamado el Problema de Valor Inicial tipo Caputo.

El estudio de la existencia y unicidad de la solución del Problema de Valor Inicial (4.3) y (4.4), como hemos dicho en la introducción, se basa en reducir este problema a una ecuación integral de Volterra de segunda especie. Esta equivalencia se muestra el siguiente teorema. Ver (cf[6])

Teorema 1 Sea $\alpha \in \mathbb{R}^{+}$. Supongamos que $g:[0, h] \times A \rightarrow \mathbb{R}, A \subset \mathbb{R}$ es continua con respecto a la variable $x$ en $[0, h]$. Una función $f \in C[a, b]$ es solución del Problema de Valor Inicial (4.3) y (4.4) si y solo si es solución de la ecuación integral del tipo Volterra

$$
f(x)=\sum_{i=0}^{n-1} \frac{b_{i}}{i !} x^{i}+\frac{1}{\Gamma(\alpha)} \int_{a}^{x}(x-t)^{\alpha-1} g(t, f(t)) d t
$$

donde $n=\lceil\alpha\rceil+1$

Demostración.

$\Rightarrow$ ) Por el Lemma 1 la ecuación (4.3) se puede escribir como

$$
\begin{aligned}
g(x, f(x)) & =D_{* 0}^{\alpha} f(x)=D^{\alpha}\left(f-T_{n-1}[f ; 0]\right)(x) \\
& =D^{n} I^{n-\alpha}\left\{f-T_{n-1}[f ; 0]\right\}(x) \\
& =D^{n} I_{0}^{n-\alpha}\left(f(x)-\sum_{i=0}^{n-1} \frac{b_{i}}{i !} x^{i}\right)
\end{aligned}
$$

Por hipotesis $g$ es continua, entonces,

$$
D^{n} I_{0}^{n-\alpha}\left(f(x)-\sum_{i=0}^{n-1} \frac{b_{i}}{i !} x^{i}\right)
$$


es continua y por lo tanto

$$
I_{0}^{n-\alpha}\left(f(x)-\sum_{i=0}^{n-1} \frac{b_{i}}{i !} x^{i}\right) \in C^{n}[a, b]
$$

Por otro lado, teniendo en cuenta (4.3) y aplicando (3.7) a

$$
\begin{gathered}
I_{0}^{n-\alpha}\left(f(x)-\sum_{i=0}^{n-1} \frac{b_{i}}{i !} x^{i}\right) \\
I_{0}^{n} g(x, f(x))=I_{0}^{n} D^{n}\left(I_{0}^{n-\alpha}\left(f(x)-\sum_{i=0}^{n-1} \frac{b_{i}}{i !} x^{i}\right)\right) \\
=I_{0}^{n-\alpha}\left(f(x)-\sum_{i=0}^{n-1} \frac{b_{i}}{i !} x^{i}\right)-\sum_{j=0}^{n-1}\left[D^{j}\left(I_{0}^{n-\alpha}\left(f(x)-\sum_{i=0}^{n-1} \frac{b_{i}}{i !} x^{i}\right)\right)\right]_{x=0} \frac{x^{j}}{j !}
\end{gathered}
$$

Por la regla de Leibitz, se tiene que

$$
D I_{0}^{n-\alpha}\left(f(x)-\sum_{i=0}^{n-1} \frac{b_{i}}{i !} x^{i}\right)=I_{0}^{n-\alpha}\left(f^{1}(x)-\sum_{i=0}^{n-1} \frac{b_{i}}{i !} x^{i-1}\right)
$$

Repitiendo este mismo procedimiento $j$ veces $(j=0,1, \ldots \ldots, n-1)$ se obtiene que

$$
\begin{aligned}
D^{j}\left(I_{0}^{n-\alpha}\left(f(x)-\sum_{i=0}^{n-1} \frac{b_{i}}{i !} x^{i}\right)\right) & =I_{0}^{n-\alpha}\left(f^{(j)}(x)-\sum_{i=j}^{n-1} \frac{b_{i}}{(i-j) !} x^{i-j}\right) \\
& =\frac{1}{\Gamma(n-\alpha)} \int_{0}^{x}(x-t)^{n-\alpha-1}\left(f^{j}(t)-\sum_{i=j}^{n-1} \frac{b_{i}}{(i-j) !} t^{i-j}\right) d t
\end{aligned}
$$

Sí en la última expresión hacemos el cambio de variable $s=\frac{t}{x}$, y llamando

$$
A=D^{j}\left(I_{0}^{n-\alpha}\left(f(x)-\sum_{i=0}^{n-1} \frac{b_{i}}{i !} x^{i}\right)\right)
$$

se tiene que

$$
\begin{aligned}
A & =\frac{1}{\Gamma(n-\alpha)} \int_{0}^{1} \underbrace{x^{n-\alpha-1}}_{c t e}(1-s)^{n-\alpha-1}\left(f^{j}(t)-\sum_{i=j}^{n-1} \frac{b_{i}}{(i-j) !}(x s)^{i-j}\right) x d s \\
& =\frac{x^{n-\alpha}}{\Gamma(n-\alpha)} \int_{0}^{1}(1-s)^{n-\alpha-1}\left(f^{j}(x s)-\sum_{i=j}^{n-1} \frac{b_{i}}{(i-j) !}(x s)^{i-j}\right) d s
\end{aligned}
$$




$$
\begin{aligned}
& \text { como } n>\alpha \Rightarrow n-\alpha>0 \\
& \lim _{x \rightarrow 0} A=\lim _{x \rightarrow 0}\left[\frac{x^{n-\alpha}}{\Gamma(n-\alpha)} \int_{0}^{1}(1-s)^{n-\alpha-1}\left(f^{j}(x s)-\sum_{i=j}^{n-1} \frac{b_{i}}{(i-j) !}(x s)^{i-j}\right) d s\right]=0
\end{aligned}
$$

Por lo tanto

$$
\lim _{x \rightarrow 0} D^{j}\left(I_{0}^{n-\alpha}\left(f(x)-\sum_{i=0}^{n-1} \frac{b_{i}}{i !} x^{i}\right)\right)=0
$$

Luego

$$
I_{0}^{n} g(x, f(x))=I_{0}^{n-\alpha}\left(f(x)-\sum_{i=0}^{n-1} \frac{b_{i}}{i !} x^{i}\right)
$$

Aplicando el operador derivada fraccionaria de Riemann-Liouville de orden $n-\alpha$ a ambos miembros de (4.8) se tiene

$$
\begin{aligned}
D_{0}^{n-\alpha} I_{0}^{n} g(x, f(x)) & =\underbrace{D_{0}^{n-\alpha} I_{0}^{n-\alpha}}_{I}\left(f(x)-\sum_{i=0}^{n-1} \frac{b_{i}}{i !} x^{i}\right) \\
& =\left(f(x)-\sum_{i=0}^{n-1} \frac{b_{i}}{i !} x^{i}\right) \\
D_{0}^{-\alpha} g(x, f(x)) & =f(x)-\sum_{i=0}^{n-1} \frac{b_{i}}{i !} x^{i}
\end{aligned}
$$

Luego, teniendo en cuenta que $D_{0}^{-\alpha}=I_{0}^{\alpha}$ y despejando $f(x)$ en (4.9) obtenemos

$$
f(x)=I_{0}^{\alpha} g(x, f(x))+\sum_{i=0}^{n-1} \frac{b_{i}}{i !} x^{i}
$$

$\Leftarrow)$ Por hipotesis se tiene que

$$
f(x)=I_{0}^{\alpha} g(x, f(x))+\sum_{i=0}^{n-1} \frac{b_{i}}{i !} x^{i}
$$


aplicando la derivada fraccionaria de Caputo a ambos miembros de (4.11) y usando la linealidad $D_{* 0}^{\alpha}$ se tiene

$$
\begin{aligned}
D_{* 0}^{\alpha} f(x) & =D_{* 0}^{\alpha}\left(I_{0}^{\alpha} g(x, f(x))+\sum_{i=0}^{n-1} \frac{b_{i}}{i !} x^{i}\right) \\
& =D_{* 0}^{\alpha}\left(\sum_{i=0}^{n-1} \frac{b_{i}}{i !} x^{i}\right)+D_{* 0}^{\alpha} I_{0}^{\alpha} g(x, f(x)) \\
& =I_{0}^{n-\alpha} \underbrace{D^{n}\left(\sum_{i=0}^{n-1} \frac{b_{i}}{i !} x^{i}\right)}_{=0}+\underbrace{D_{* 0}^{\alpha} I_{0}^{\alpha}}_{I} g(x, f(x)) \\
& =g(x, f(x))
\end{aligned}
$$

Para obtener las condiciones iniciales, derivemos $i$ veces (4.5). En efecto

$$
\begin{aligned}
f^{(i)}(x) & =D^{(i)}\left(\sum_{j=0}^{n-1} \frac{b_{j}}{j !} x^{j}\right)+D^{(i)}\left(\frac{1}{\Gamma(\alpha)} \int_{0}^{x}(x-t)^{\alpha-1} g(t, f(t))\right) d t \\
& =D^{(i)}\left(\sum_{j=0}^{n-1} \frac{b_{j}}{j !} x^{j}\right)+\underbrace{D^{(i)} I_{0}^{\alpha}}_{i \leq \alpha \Rightarrow i-\alpha<0} g(x, f(x)) \\
& =\sum_{j=i}^{n-1} \frac{b_{j}}{(j-i) !} x^{j-i}+\underbrace{D^{i-\alpha}}_{I^{\alpha-i}} g(x, f(x)) \\
& =\sum_{j=i}^{n-1} \frac{b_{j}}{(j-i) !} x^{j-i}+I_{0}^{\alpha-i} g(x, f(x)) \\
& =\sum_{j=i}^{n-1} \frac{b_{j}}{(j-i) !} x^{j-i}+\frac{1}{\Gamma(\alpha-i)} \int_{0}^{x}(x-t)^{\alpha-i-1} g(t, f(t)) d t \\
& : t^{n}=s x \Rightarrow d t=x d s y(x-t)=x(1-s) \\
& =\sum_{j=i}^{n-1} \frac{b_{j}}{(j-i) !} x^{j-i}+\frac{1}{\Gamma(\alpha-i)} \int_{0}^{1} x^{\alpha-i-1}(1-s)^{\alpha-i-1} g(s, f(s)) x d s \\
& =\sum_{j=i}^{n-1} \frac{b_{j}}{(j-i) !} x^{j-i}+\frac{x^{\alpha-i}}{\Gamma(\alpha-i)} \int_{0}^{1}(1-s)^{\alpha-i-1} g(s, f(s)) d s \\
& =b_{i}+\sum_{j=i+1}^{n-1} \frac{b_{j}}{(j-i) !} x^{j-i}+\frac{x^{\alpha-i}}{\Gamma(\alpha-i)} \int_{0}^{1}(1-s)^{\alpha-i-1} g(s, f(s)) d s
\end{aligned}
$$

uego

$$
f^{i}(0)=b_{i}(i=0,1, . ., n-1)
$$


Ejemplo 1 Teniendo en cuenta la definición 8 , con $\alpha>0$ y $k=1,2, \ldots, n-1$, consideremos el siguiente Problema de Valor Inicial.

$$
\begin{array}{r}
D_{* 0}^{\alpha} y(x)=y(x) \\
y(0)=1 \\
y^{k}(0)=b_{k}
\end{array}
$$

siendo $g(x, y(x))=y(x)$, satisface las conciones del Teorema 1, por lo que existe una única función continua que verifica el Problema de Valor Inicial. La ecuación integral correspondiente a este problema es

$$
y(x)=1+\frac{1}{\Gamma(\alpha)} \int_{0}^{x}(x-t)^{\alpha-1} y(t) d t
$$

y las iteraciones de Picard vienen dadas por

$$
y_{n}(x)=1+\frac{1}{\Gamma(\alpha)} \int_{0}^{x}(x-t)^{\alpha-1} y_{n-1}(t) d t \quad n \in \mathbb{N} \text { con } y_{0}(x)=1
$$

Es decir

$$
\begin{gathered}
y_{1}(x)=1+\frac{1}{\Gamma(\alpha)} \int_{0}^{x}(x-t)^{\alpha-1} d t=1+\frac{1}{\Gamma(\alpha+1)} x^{\alpha} \\
y_{2}(x)=1+\frac{1}{\Gamma(\alpha)} \int_{0}^{x}(x-t)^{\alpha-1} y_{1}(t) d t=1+\frac{1}{\Gamma(\alpha+1)} x^{\alpha}+\frac{1}{\Gamma(2 \alpha+1)} x^{2 \alpha}
\end{gathered}
$$

En general se tiene que:

$$
\begin{aligned}
y_{n}(x) & =1+\frac{1}{\Gamma(\alpha+1)} x^{\alpha}+\frac{1}{\Gamma(2 \alpha+1)} x^{2 \alpha}+\ldots+\frac{1}{\Gamma n \alpha+1} x^{n \alpha} \\
& =\sum_{i=0}^{n} \frac{\left(x^{\alpha}\right)^{i}}{\Gamma(i \alpha+1)}
\end{aligned}
$$

Por lo tanto

$$
y(x)=\lim _{n \rightarrow \infty} y_{n}(x)=\sum_{i=0}^{\infty} \frac{\left(x^{\alpha}\right)^{i}}{\Gamma(i \alpha+1)}=E_{\alpha}\left(x^{\alpha}\right)
$$

Luego, $y(x)=E_{\alpha}\left(x^{\alpha}\right)$, que es la función de Mittag-Leffler, es solución del Problema de Valor Inicial. 


\section{Concluciones}

A lo largo de este trabajo hemos dado las condiciones de existencia y unicidad de la solución para una ecuaciones diferenciales de orden fraccionario, lo cual es considerado como una generalización del Teorema de Picard para ecuaciones diferenciales de orden entero. Además hemos usado la derivada fraccionaria de Caputo y la equivalencia con las ecuaciones integrales de Volterra de segunda especie. Por lo tanto, se concluye que:

1. Las ecuaciones diferenciales de orden fraccionario son una generalización de las ecuaciones diferenciales de orden entero, es decir tiene la particularidad de permitir abordar fenomenos diferentes de los cuales las ecuaciones diferenciales que los describen son casos particulares del orden fracionario de la ecuación original. Como hemos visto en el ejemplo, si tomamos $\alpha=2$

2. La equivalencia entre las ecuaciones diferenciales de orden fraccionario con las ecuaciones integrales de Volterra de segunda especie fué fundamental para el desarrollo de este trabajo.

3. Las condiciones de existencia y unicidad del Problema de Valor Inicial (4.3) y (4.4) son similares a las condiciones de existencia y unicidad en ecuaciones diferenciales de orden entero.

En efecto: Si $g:[0, h] \times A \rightarrow \mathbb{R}$ continua en $A \subseteq \mathbb{R}$, entonces sabemos que existe al menos una función que es solución y si además le pedimos que $g:[0, h] \times A \rightarrow \mathbb{R}$ sastifaga la condición de Lipschitz con respecto a la segunda variable, la solución es única. (cf.[2] teorema 6 página 185)

\section{Referencias}

[1] Antón, Lombardero Ozores. Cálculo fraccionario y Dinámica Newtoniana. Pensamiento Matemático. Volumen IV. 2014.

[2] Birkhoff, Garrett.; Rota, Gian-Carlo. Ordinary Differential Equations 4th Edition. John Wiley \& Sons, New Jersey. 1989.

[3] Burgos J. Cálculo infinitesimal de una variable. MacGraw-Hill. 1996

[4] Fava,N; Zo. Medida e Integral de Lebesgue. Red Olímpica. 1996.

[5] F.G. Tricomi. Integral Equations. Dover Publications.1985

[6] Kilbas, A. A.; Srivastava, H. M.; and Trujillo, J. J. Theory and Applications of Fractional Differential Equations; Amsterdam, Netherlands, Elsevier. 2006.

[7] Miana, P. Convolution Products in $L^{1}\left(\mathbb{R}^{+}\right)$, integral transforms and fractional calculus. Fraccional Calculus and Appied Analisis.Vol 8. Nro 4. 2005. 
[8] Podlubny, I.. Fractional Differential Equations. San Diego Academic Press. 1999

[9] Podlubny, K, Despotovic, V, Skovranekt, McNaughton,B.Shadows on the Walls Geometric Interpretations of Fractional Integration. The Journal of Online mathematics and Its Applications. Vol 7. 2007

[10] Samko, S.; Kilbas, A.A.; and Marichev, O. Fractional Integrals and Derivatives. Theory and Applications. Langhorne, PA: Gordon and Breach Science Publishers. 1993 